\title{
Aerobic Bacteriological Profile of Chronic Suppurative Otitis Media and Special Reference to Methicillin Resistant Staphylococcus aureus
}

\author{
Jyoti R. Hundekar*, Surekha, Y.A. Mariraj Jeer and S. Krishna \\ Department of Microbiology, Vijayanagara Institute of Medical Sciences, Bellary, India \\ *Corresponding author
}

\begin{tabular}{|l|}
\hline Key w or d s \\
$\begin{array}{l}\text { Bacteriological } \\
\text { profile, Chronic } \\
\text { suppurative otitis } \\
\text { media. }\end{array}$ \\
\hline Article Info \\
\hline $\begin{array}{l}\text { Accepted: } \\
\text { 17 June } 2019 \\
\text { Available Online: } \\
\text { 10 July } 2019\end{array}$ \\
\hline
\end{tabular}

\section{Introduction}

Otitis media has long been recognized as a common disease with its sequele. Hippocrates described the disease in $450 \mathrm{BC}^{1}$. Incidence of this disease is higher in developing countries especially among low socioeconomic society because of malnutrition, overcrowding, poor hygiene,
Chronic Suppurative Otitis Media (CSOM) is a well known disease for its persistence and recurrence in spite of treatment. It was found to be the single major cause of conductive deafness, speech disorders. It involves considerable morbidity and can cause extra and intracranial complications. Due to development of beta lactamase producing microorganisms, drug resistant strains emerge making drug resistance a major cause of treatment failure. The isolation of Methicillin Resistant Staphylococcus aureus (MRSA) was reported within 1year of introduction of methicillin. In many hospitals $40-50 \%$ of Staphylococcus aureus isolates are now resistant to methicillin. Out of 129 ear discharge samples, 86 samples showed growth, the isolates were identified by conventional microbiological methods including colony morphology and standard biochemical reactions. Antibiotic susceptibility tests were performed by Kirby-Bauer disk diffusion method. MRSA in isolated $S$. aureus was detected by cefoxitin disk diffusion method. The MIC(Minimum inhibitory concentration) was determined by agar dilution method and Etest. Among 129 ear samples, 100 bacterial isolates were isolated, the most common organism isolated was Staphylococcus aureus 36 (36\%) followed by Pseudomonas spp. 34 (34\%), Coagulase negative Staphylococcus 9(9\%). Others include, E. coli, Acinetobacter spp, Citrobacter spp, Streptococcus spp (4\%) each. Klebsiella spp and Proteus vulgaris 2(2\%) each was isolated and Enterobacter spp, in one sample (1\%) isolated. The antibiotic susceptibility testing showed Amikacin as the most effective drug followed by, gentamicin, cefotaxime, cotrimoxazol, ciprofloxacin and amoxyclav. 
The reported overall extracranial and intracranial complication rate in CSOM varies from $0.7 \%$ to $3.2 \%$; extracranial complications alone from $0.5 \%$ to $1.4 \%$ and intracranial complications from $0.3 \%$ to $2.0 \%$.Such complications range from persistant otorrhoea, mastoiditis, labyrinthitis, and facial nerve paralysis to more serious intracranial abscess or thromboses ${ }^{4}$.

CSOM is a disease of multiple etiologies and is well known for its persistence and recurrence in spite of treatment. Incidence of CSOM increasing in the past $10-20$ years. It was found to be the single major cause for conductive deafness $(66.3 \%)$ and is also responsible for $1.5 \%$ of speech disorders ${ }^{5}$. Hearing loss and ear diseases in India according to WHO, CSOM accounts about $5.2 \%{ }^{6}$.

CSOM has received considerable attention, not only because of its high incidence and chronicity, but also because of issues such as bacterial resistance and ototoxicity with both topical and systemic antibiotics. The wide spread use of antibiotics has precipitated the emergence of multiple resistant strains of bacteria which can produce both primary and post-operative ear infections ${ }^{7}$.

The indiscriminate, haphazard and hay hearted use of antibiotics and the poor follow up of the patients have resulted in the persistence of low grade infections ${ }^{3}$.

Due to development of beta lactamase producing microorganisms, drug resistant strains emerge making drug resistance a major cause of treatment failure in these patients $^{8}$. The isolation of Methicillin Resistant Staphylococcus aureus (MRSA) was reported within 1year of introduction of methicillin. The prevalence of MRSA has since increased steadily. In many hospitals 40-50\% of Staphylococcus aureus isolates are now resistant to methicillin ${ }^{9}$. As the proper selection of antibiotics helps in preventing drug resistance and also clearing of infection ${ }^{5}$.

Identification and detection of MRSA and ESBL producers is also important before treatment of CSOM cases. As cases with these infections are resistant to routine Betalactum antibiotics and penicillin. Knowledge of local microorganism pattern and their antibiotic sensitivity pattern is essential for effective low cost treatment ${ }^{3}$.

\section{Objectives}

1. Isolation of aerobic bacteria from various CSOM samples.

2. To know the Antibiotic sensitivity pattern of isolated bacteria.

3. Detection of MRSA in isolated Staphylococcus aureus.

\section{Materials and Methods}

The present study "Aerobic bacteriological profile of chronic suppurative otitis media and special reference to methicillin resistant staphylococcus aureus." was conducted in the Department of Microbiology, Vijayanagar institute of medical sciences, Ballari, from 2015 to 2016. 129 samples with CSOM of all age groups and both sexes attending outpatient department and those admitted in ENT wards were selected randomly for the study.

Ear discharges were collected by two sterile swabs under aseptic precautions in clinically diagnosed cases of CSOM attending ENT out patient department. One swab for Gram staining and other one for aerobic culture, plating on blood agar and Macconkey's agar and incubating at $37^{\circ}$ for an 18-24 hours.

Total of 109 cases were studied, from which 129 samples were collected and processed. When there was growth, the isolates were identified by conventional microbiological 
methods including colony morphology and standard biochemical reactions. Antibiotic susceptibility tests were performed by KirbyBauer disk diffusion method, according to the guidelines of Clinical and Laboratory Standards Institute (CLSI, M100-S12 document).

MRSA in isolated S.aureus was detected by cefoxitin disk diffusion method. The MIC (Minimum inhibitory concentration) was determined by agar dilution method and Etest.

\section{Results and Discussion}

Out of 129 samples, 86 samples showed culture positivity, in which 100 were of bacterial isolates.

Out of 109 cases, 38(35\%) cases were from urban areas and $71(65 \%)$ were from rural areas
Out of 109 cases, 70 cases were males, 39 cases were females. Incidence of CSOM was maximum in the age group of 0-10 years in which 20(29\%) were males and 11(28\%) were females. As the age increased, incidence of CSOM decreased.

Isolation of bacteria was maximum in 0-20 years age group and as age increases the percentage of isolation of bacteria decreases. The culture negative was high in $0-10$ and $>40$ years of age groups (Table 1).

Out of 109 cases, $38(35 \%)$ cases were from urban areas and $71(65 \%)$ were from rural areas.

Table 2 shows that unilateral infection is more common compared to bilateral infection. Right ear infected was in 35(32\%) cases, left ear involved in 54(50\%) cases and bilateral infection was in 20(18\%) cases.

Table.1 Age-sex wise distribution of study subjects

\begin{tabular}{|c|c|c|c|c|c|c|}
\hline \multirow[t]{3}{*}{ Age Group } & \multicolumn{4}{|l|}{ Sex } & \multirow{2}{*}{\multicolumn{2}{|c|}{ Total }} \\
\hline & \multicolumn{2}{|l|}{ Male } & \multicolumn{2}{|c|}{ Female } & & \\
\hline & Num & $\%$ & Num & $\%$ & Num & $\%$ \\
\hline 0 -10 years & 20 & 28.6 & 11 & 28.2 & 31 & 28.4 \\
\hline 11 - 20 years & 19 & 27.1 & 8 & 20.5 & 27 & 24.8 \\
\hline 21 - 30 years & 14 & 20 & 7 & 17.9 & 21 & 19.3 \\
\hline 31 - 40 years & 7 & 10 & 3 & 7.7 & 10 & 9.2 \\
\hline$>40$ years & 10 & 14.3 & 10 & 25.6 & 20 & 18.3 \\
\hline Total & 70 & 100 & 39 & 100 & 109 & 100 \\
\hline
\end{tabular}

Table.2 Distribution of patients based on ear involved

\begin{tabular}{|l|l|l|}
\hline Ear & Frequency & Percentage (\%) \\
\hline Right & 35 & 32 \\
\hline Left & 54 & 50 \\
\hline Bilateral & 20 & 18 \\
\hline Total & 109 & 100 \\
\hline \multicolumn{2}{r}{ Chi square -15.982 df- 2 p-value-0.00034 }
\end{tabular}


Table.3 Pattern of organisms

\begin{tabular}{|l|c|c|}
\hline Organisms & Frequency & Percentage (\%) \\
\hline Staphylococcus aureus & 36 & 36 \\
\hline Pseudomonas aeruginosa & 34 & 34 \\
\hline Citrobacter freundii & 4 & 4 \\
\hline Coagulase negative staphylococci & 9 & 9 \\
\hline E.coli & 4 & 4 \\
\hline Klebsiella spp & 2 & 2 \\
\hline Proteus Mirabilis & 2 & 2 \\
\hline Acinetobacter spp. & 4 & 4 \\
\hline Streptococcus $\boldsymbol{s p p}$ & 4 & 4 \\
\hline Enterobacter $\boldsymbol{s p p}$ & 1 & 1 \\
\hline Total & 100 & 100 \\
\hline
\end{tabular}

Chi square-160.6 df-9 p-value- 0

Table.4 Distribution based on antibiotic sensitivity

\begin{tabular}{|l|l|l|l|l|l|l|}
\hline \multirow{2}{*}{ Antibiotics } & \multicolumn{3}{l|}{ Sensitivity } & \multicolumn{3}{l|}{ Total } \\
\cline { 2 - 7 } & \multicolumn{2}{|l|}{ Resistance } & \multicolumn{2}{l|}{ Sensitive } & & \\
\cline { 2 - 6 } & Num & $\mathbf{\%}$ & Num & $\mathbf{\%}$ & Num & $\%$ \\
\hline Amikacin & 27 & 27 & 73 & 73 & 100 & 100.0 \\
\hline Ciprofloxacin & 67 & 67 & 33 & 33 & 100 & 100.0 \\
\hline Amoxyclav & 79 & 79 & 21 & 21 & 100 & 100.0 \\
\hline Gentamycin & 53 & 53 & 47 & 47 & 100 & 100.0 \\
\hline Cefotoxime & 54 & 54 & 46 & 46 & 100 & 100.0 \\
\hline Co trimaxazole & 52 & 52 & 48 & 48 & 100 & 100.0 \\
\hline
\end{tabular}

Table.5 Determination of MIC by agar dilution and E test methods

\begin{tabular}{|l|l|l|l|l|l|}
\hline $\begin{array}{l}\text { Test: agar } \\
\text { dilution }(\boldsymbol{\mu g} / \mathbf{m L})\end{array}$ & $\mathbf{6}$ & $\mathbf{1 2}$ & $\mathbf{2 4}$ & $\mathbf{4 8}$ & total \\
\hline Isolates & 2 & 3 & 3 & 0 & 8 \\
\hline \multicolumn{5}{|c|}{ MIC standard $(\mu \mathrm{g} / \mathrm{mL})$ - Susceptible; $\leq 2$} & Resistant- $\geq 4$ \\
\hline
\end{tabular}

\begin{tabular}{|l|l|l|l|l|l|l|}
\hline $\begin{array}{l}\text { Test: E-test } \\
(\boldsymbol{\mu g} / \mathbf{m L})\end{array}$ & $\mathbf{1 2}$ & $\mathbf{1 6}$ & $\mathbf{2 4}$ & $\mathbf{3 2}$ & $\mathbf{4 8}$ & Total \\
\hline Isolates & 2 & 0 & 3 & 0 & 3 & 8 \\
\hline
\end{tabular}

Graph.1 Distribution of patients based on area 


\section{DISTRDISTRIBUTION OF MSSA AND MRSA AMONGST STAPHYLOCOCCUS AUREUS}

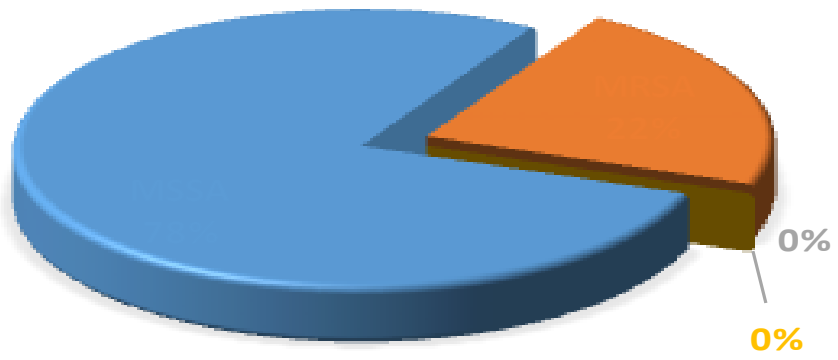

Out of 129 ear samples, 86 samples showed culture positivity, in which 100 were of bacterial isolates, the most common organism isolated was Staphylococcus aureus 36 (36\%) followed by Pseudomonas spp. 34 (34\%), Coagulase negative Staphylococcus 9(9\%). Others include, E. coli, Acinetobacter spp, Citrobacter spp, Streptococcus spp 4(4\%) each. Klebsiella spp and Proteus vulgaris $2(2 \%)$ each was isolated and Enterobacter spp, in one sample (1\%) isolated. Both children and adults most common bacteria were Staphylococcus aureus and Pseudomonas spp. In the adults Staphylococcus aureus was the common infecting organism (Table 3).

The antibiotic susceptibility testing showed Amikacin as the most effective drug followed by, gentamicin, cefotaxime, cotrimoxazol, ciprofloxacin and amoxyclav.

Among 100 isolates, $36(36 \%)$ were of Staphylococcus aureus. In 36 isolates 28 
(78\%) isolates were MSSA while 22(22\%) were MRSA. MRSA isolates showed $75 \%$ resistance to ampicillin, $75 \%$ resistance to gentamycin, $37.5 \%$ resistance to clindamycin and $12.5 \%$ resistance to erythromycin and the MSSA isolates showed $60.7 \%$ resistance to ampicillin, $28.6 \%$ resistance to erythromycin, $32.1 \%$ resistance to gentamycin, $14.3 \%$ resistance to clindamycin and both MRSA and MSSA isolates were $100 \%$ sensitive to vancomycin and linezolide.

MIC detection by agar dilution method showed MIC of $12 \mu \mathrm{g} / \mathrm{mL}, 24 \mu \mathrm{g} / \mathrm{mL}$ for 3 isolates each. and 2 isolates MIC was 6 $\mu \mathrm{g} / \mathrm{mL}$.

In E test MIC of $24 \mu \mathrm{g} / \mathrm{mL}, 48 \mu \mathrm{g} / \mathrm{mL}$ for 3 isolates each and 2 isolates MIC was 12 $\mu \mathrm{g} / \mathrm{mL}$.

In the present study the maximum number of patients was in the age group of 0 to 10 years $31(28.4 \%)$. Which is correlating with study by Adoga et al., and Geeta et al., In Adoga et $a l$. , in a total of 80 patients $24(30 \%)$ children were below 10 years of age ${ }^{10}$ and study by Geeta et al., out of 250 samples, $(30.4 \%)^{8}$ were of $0-10$ years of age group. These studies correlate with present study (Table 4 and 5).

Present study showed 70(64.2\%) were males and $39(35.7 \%)$ were females. Our study correlates with the studies by Swarooparani et al., studied 99 patients with CSOM and observed that males $(56.6 \%)$ were more commonly affected than females $(43.4 \%)^{5}$. In a study by Sanjay Kumar et al., out of 62 patients, 38(61.29\%) were males and $24(38.7 \%)$ were female patients ${ }^{11}$.Vijay Kumar Poorey et al., studied 120 patients of CSOM in which 68(56.66\%) were males and $52(43.34 \%)$ were females ${ }^{2}$. Study by Harshika et al., included 130 patients, in them male predominance $(53.07 \%)$ in occurrence of CSOM when compared to females
$(46.92 \%)^{12}$. And in a study by Nikakhlagh et al., found male predominance $(54 \%)$ than female $(46 \%)^{13}$. These studies correlate with the present study. The male predominance may be because of their more exposed way of life.

In the present study incidence of CSOM cases was more common in rural area $(66 \%)$, as compared to urban area (34\%).

Ashib Parvez et al., conducted a study in a total of 630 children $6.1 \%$ CSOM cases were seen, where they found $7 \%$ of them from rural areas and $1.8 \%$ were from urban areas. It was found to associate with nutritional status of child and standard of living index ${ }^{14}$.

And in a study by Arti Agrawal et al., studied 125 patients out of which $70(56.0 \%)$ were from rural and 55(44.0\%) from urban citations $^{15}$. This study correlated with our study.

In the present study Staphylococcus aureus and Pseudomonas spp were the most common infecting organisms in both children and in adults. This finding correlated with Geeta et $a l^{8}$ and Swarooparani et $a l^{5}$.

In the present study unilateral infection $(82 \%)$ was more common compared to bilateral infection (18\%). Swarooparani et al., studied 80 patients of CSOM and reported $76.8 \%$ unilateral and $23 \%$ in bilateral ${ }^{5}$. And Sanjay Kumar et al., studied 62 patients, among them $54(87.09 \%)$ patients had unilateral disease, while $8(12.09 \%)$ patients had bilateral disease $^{11}$. These findings are consistent with the present study.

A total of 100 bacterial isolates were isolated from86 culture positive cases. The most common organism isolated was Staphylococcus aureus 36 (36\%) followed by Pseudomonas spp. 34 (34\%), Coagulase negative Staphylococcus 9(9\%). Others 
include, E. coli, Acinetobacter spp, Citrobacter spp, Streptococcus spp 4(4\%) each. Klebsiella spp and Proteus vulgaris $2(2 \%)$ each was isolated and Enterobacter spp, in one sample (1\%) isolated.

Pseudomonas spp was the predominant bacteria isolated from the patients with CSOM in studies reported by Kenna et al., ${ }^{16}$, Fliss et al., ${ }^{17}$, Papastavros et al.., ${ }^{18}$, Arguedas et al., ${ }^{19}$ and Brook ${ }^{20}$ et al.

Staphylococcus aureus was the predominant bacteria isolated from the patients with CSOM in studies reported by Nikakhalagh et al., ${ }^{13}$, De uzeda et al., ${ }^{21}$ and Miro et al., ${ }^{22}$. This study observation correlates with the above studies.

In our study, the sensitivity of Staphylococcus aureus to aminoglycosides like amikacin and gentamicin were $69.4 \%$, 55.5\% respectively. flouroquinolones like ciprofloxacin showed $22 \%$ of sensitivity and for cephalosporins like cefotaxime, ceftriaxone showed $52.7 \%$, and $33.3 \%$ sensitivity respectively. MRSA showed sensitive to doxycycline $87.5 \%$, clindamycin 62.5\%, Amikacin 50\%, gentamycin $25 \%$, cefotaxim $50 \%$ and showed resistance to ampicillin.

Pseudomonas spp showed sensitivity to amikacin 64.7\%, gentamycin $35.3 \%$, ciprofloxacin $32.3 \%$ and cephalosporins like cefotaxime $29.4 \%$, ceftriaxone $50 \%$.

However sensitivity pattern in other studies varied.

High level of sensitivity to aminoglycosides were seen in a study by Sanjana et al., reported that, Pseudomonas spp was the most common isolate, in which $93.2 \%$ isolates were sensitive to tobramycin, $91.5 \% \%$ sensitive to ceftazidime and $77.9 \%$ to amikacin, for ciprofloxacin $50.8 \%$ and $25.4 \%$ for gentamycin sensitivity was seen.
Staphylococcus aureus (other than MRSA) showed $95.2 \%$ sensitive to cloxacillin, $83.3 \%$ to gentamycin and $78.5 \%$ to erythromycin. Only $7.1 \%$ were sensitive to ampicillin and $26.1 \%$ to ciprofloxacin. More than $90 \%$ of pseudomonas and $S$. aureus were sensitive to tobramycin and cloxacillin respectively ${ }^{3}$.

Rajat Prakash et al., in their study observed most common organism was $S$. aureus fallowed by Pseudomonas spp. Antimicrobial profile of aerobic isolates revealed maximum sensitivity to amikacin (95.5\%), ceftriaxone $(83.4 \%)$ and gentamycin $(82.7 \%)^{23}$.

Study conducted by Nikakhlagh et al., S. aureus showed $100 \%$ sensitivity to ceftazidime $87.5 \%$ for cefotaxime and $66.5 \%$ for amikacin. All of the pseudomonas were resistant cephalosporins like to cefotaxime and $81.2 \%$ of them were resistant to ceftazidime $^{13}$.

Study done by Swarooparani et al., revealed s.aureus was sensitive to gentamycin $(72.4 \%)$, amoxyclav $66.5 \%$ and ciprofloxacin $44.8 \%$. Pseudomonas spp and other gram negative bacteria were sensitive to amikacin $70-100 \%$, cefotaxime $50-100 \%$, amoxyclav $50-100 \%$. MRSA showed sensitivity to gentamycin $41.7 \%$, and amoxyclav $41.7 \%$ but resistant to ampicillin, erythromycin and ceftazidim ${ }^{5}$.This study is correlating with our study.

In the present study flouroquinolones $22 \%$ and cephalosporin group $43 \%$ showed sensitivity for $S$. aureus.

For Pseudomonas spp to quinolone and cephalosporin groups showed $39.7 \%$ and $32.3 \%$ sensitivity respectively, which shows for quinolone and cephalosporin family there is appearance of antibiotic resistant strains of S.aureus and Pseudomonas spp which is a matter of great concern.

Other isolates in the present study showed 
high sensitivity to aminoglycosides like amikacin (73\%), and low sensitive to ciprofloxacin (33\%). This study correlates with the studies reported by Sanjana et al., ${ }^{3}$ Nikakhlagh et al., ${ }^{13}$ and Swarooparani et al., .

In conclusion the aerobic bacteriological study of CSOM showed Staphylococcus aureus as the most common causative agent followed by Pseudomonas aeruginosa, Coagulase negative Staphylococcus, Citrobacter spp, Klebsiella spp, Proteus mirabilis and E.coli.

The antibiotic susceptibility testing showed Amikacin as the most effective drug followed by, gentamicin, cefotaxime and cotrimoxazol, ciprofloxacin, amoxyclav. Carefully selected local and/or systemic antibiotics guided by culture and sensitivity, along with the use of frequent ear toilet is an effective treatment modality. This will prevent development of drug resistance and administration of unwanted antibiotics.

\section{References}

1. Gerald B. Healy Otitis media and middle effusions. Otorhinolaryngology, Head and Neck Surgery. John Jacob Ballenger. $17^{\text {th }}$ edition, Williams and Wilkins 2009. 17-27, 217-226, 229-238.

2. Vijay Kumar Poorey, Pooja Thakur. Clinicomicrobiological evaluation and antibiotic susceptibility in cases of chronic suppurative otitis media. Indian Journal of Otology. April 2015; Vol 21; Issue 2:107-110.

3. R K. Sanjana, Y.I. Singh N.S. Reddy. Aerobic bacteriology of chronic suppurative ottis media (CSOM) in tertiary care hospital: A retrospective study. Journal of college of Medical sciences-Nepal. 2011; 7: p. 1-8.

4. Monique Verhoeff, Erwin L. van der veen, Maroeska M. Rovers, Elisabeth
A.M. Sanders, Anne G.M. Schilder. Chronic suppurative otitis media: A review. International journal of pediatric otorhinolaryngiology. 2006;: p.1-12.

5. Swarooprani NB, Kardesai SG and Metgud SC. Aerobic bacteriological study of Chronic suppurative otitis media with special reference to MRSA and ESBL. SMU Medical Journal. 2014 January; 1: p. 119-128.

6. Saurabh Varshney. Deafness in India. Indian Journal of Otology. April 2016; Vol 22; Issue 2:73-76.

7. Sateesh Kumar Malkappa,Saileela Kondapaneni et al., Study of aerobic bacteriological isolates and their antibiotic susceptibility pattern in chronic suppurative otitis media. Indian Journal of Otology. July 2012; Vol 18; Issue 3:136-139.

8. S.H, Geeta. "Study of aerobes, Anaerobes \& Fungi in CSOM in a referral Hospital of Bangalore Rural". Journal of Evaluation of medical and Dental Sciences. 2014 June 09; 3(23): p. 6297-6303.

9. Mark C. Enright, D. Ashley Robinson, Gaynor Randle et al. The evolutionary history of methicillin-resistant Staphylococcus aureus (MRSA). PNAS; May 28, 2002; vol. 99; no. 11: 76877692.

10. AS Adoga, EN Ma`an, D Malu et al. Swab and aspiration specimen collection methods and antibiogram in chronic suppurative otitis media at Jos University Teaching Hospital: Which is superior? Annals of African Medicine. 2010; Volume 9(4): 230-234.

11. Sanjay Kumar, Ravinder Sharma et al. Bacterial flora of infected unsafe CSOM. Indian Journal of Otology. October 2012; 18(4): 208-211.

12. Y. K. Harshika, S. Sangeetha and R. Prakash. Microbiological Profile of CSOM and their Antibiotic Sensitivity 
Pattern in a Tertiary Care Hospital. Int.J.Curr.Microbiol.App.Sci (2015) 4(12): 735-743.

13. S. Nikakhlagh, A. D. Khoskravi, A. Fazlipour, M. Safarzadeh and N Rashidi. Microbiological findings in patients with chronic suppurative otitis media. J.Med.Sci. 2008 August 01; 8(5): 503506.

14. AshibParvez, Zulfia Khan, SF Hashmi et al. A cross sectional study of chronic suppurative otitis media and its associated factors among primary school children in rural and urban areas of Aligarh, India. Int $\mathbf{J}$ Community Med Public Health. 2016 Aug; 3(8): 21502154.

15. Arti Agrawal, Dharmendra Kumar, Ankur Goyal et al. Microbiological profile and their antimicrobial sensitivity pattern in patients of otitis media with ear discharge. Indian Journal of Otology. January 2013; Vol 19; Issue 1:5-8.

16. M.A. Kenna, C.D. Bluestone, J.S. Reilly, R.P. Lusk, Medical management of chronic suppurative otitis media without cholesteatoma in children, Laryngoscope 96 (2) (1986) 146-151.

17. D.M. Fliss, R. Dagan, N. Meidan, A. Leiberman, Aerobic bacteriology of chronic suppurative otitis media without cholesteatoma in children, Ann. Otol. Rhinol. Laryngol. 101 (10) (1992) 866869.
18. T. Papastavros, H. Giamarellou, S. Varlejides, Role of aerobic and anaerobic microorganisms in chronic suppurative otitis media, Laryngoscope 96 (4) (1986) 438-442.

19. A. Arguedas, C. Loaiza, J.F. Herrera, E. Mohs, Antimicrobial therapy for children with chronic suppurative otitis media without cholesteatoma, Pediatr. Infect. Dis. J. 13 (10) (1994) 878-882.

20. I. Brook, Prevalence of beta-lactamaseproducing bacteria in chronic suppurative otitismedia, Am.J.Dis.Child, 139(3) (1985) 280-283.

21. M. de Uzeda, E.R. Rocha, Bacteriology of chronic otitis media affecting children living in Rio de Janeiro, Ear Nose Throat J. 68 (6) (1989), 448, 450, 452.

22. N. Miro, Controlled multicenter study on chronic suppurative otitis media treated with topical applications of ciprofloxacin $0.2 \%$ solution in single-dose containers or combination of polymyxin $\mathrm{B}$, neomycin, and hydrocortisone suspension, Otolaryngol. Head Neck Surg. 123 (5) (2000) 617-623.

23. Rajat Prakash, Deepak Juyal, Vikrant Negi, Shekhar Pal, Shamanthadekhandi, Munesh Sharma, and Neelam Sharma. Microbiology of chronic suppurative otitis media in tertiary care set up of Uttarkhand state, India. North American Journal of Medical Sciences. 2013 April; p. 282-287.

\section{How to cite this article:}

Jyoti R. Hundekar, Surekha, Y.A. Mariraj Jeer and Krishna, S. 2019. Aerobic Bacteriological Profile of Chronic Suppurative Otitis Media and Special Reference to Methicillin Resistant Staphylococcus aureus. Int.J.Curr.Microbiol.App.Sci. 8(07): 2135-2143. doi: https://doi.org/10.20546/ijcmas.2019.807.257 